\title{
ESTUDO DA RADIAÇÃO SOLAR GLOBAL E DO ÍNDICE DE TRANSMISSIVIDADE (KT), EXTERNO E INTERNO, EM UMA FLORESTA DE MANGUE EM ALAGOAS - BRASIL
}

\author{
CARLOS ALEXANDRE SANTOS QUERINO ${ }^{1}$, MARCOS ANTONIO LIMA MOURA ${ }^{2}$, JULIANE KAYSE \\ ALBUQUERQUE DA SILVA QUERINO ${ }^{1}$, CELSO VON RADOW $^{\mathbf{3}}$ E ARI DE OLIVEIRA MARQUES FILHO $^{4}$
}

\author{
${ }^{1}$ Universidade Federal do Amazonas (UFAM), Humaitá, AM, Brasil. \\ ${ }^{2}$ Universidade Federal de Alagoas (UFAL), Maceió, AL, Brasil. \\ ${ }^{3}$ Instituto Nacional de Pesquisas Espaciais/Centro de Ciências do Sistema Terrestre (INPE/CCST), São José \\ dos Campos, SP, Brasil. \\ ${ }^{4}$ Instituto Nacional de Pesquisa da Amazônia (INPA), Manaus, AM, Brasil. \\ casquerino@ufam.edu.br,malm@pq.cnpq.br,juliane_querino@ufam.edu.br, \\ celso.vonrandow@inpe.br, ari@inpa.gov.br
}

Recebido Março 2008 - Aceito Outubro 2010

\begin{abstract}
RESUMO
As florestas de manguezais são de extrema importância social, econômica e ambiental, pois as mesmas são detentoras de uma rica fauna e flora. O Brasil possui uma das maiores áreas de manguezais do mundo que se estende desde o extremo Norte até o Sul do país, e devido a isso, os efeitos da alteração de manguezal em área degradada influenciam diretamente no microclima da região. No aspecto meteorológico, é necessário que seja limitada a forma de exploração desse ecossistema, pois a conversão de florestas de mangues em áreas degradadas expõe a superfície à radiação solar direta alterando o balanço radiativo. Então, conhecer o comportamento da Radiação Solar Global $(\mathrm{Rg})$ e suas componentes, Radiação Difusa $(\mathrm{Rd})$ e Radiação Direta $\left(\mathrm{R}_{\mathrm{D}}\right)$, dentro das florestas, são fatores primordiais para o entendimento da disponibilidade de energia para os diversos processos desse sistema. O principal objetivo deste trabalho foi avaliar e comparar o comportamento da Rg e suas componentes $\mathrm{R}_{\mathrm{D}}$ e Rd, dentro e acima de uma floresta de mangue. As medidas de radiação solar foram provenientes de uma torre micrometeorológica, instalada dentro de uma floresta de mangue, localizada no município de Marechal Deodoro (distante $15 \mathrm{~km}$ da cidade de Maceió-AL), em uma Área de Proteção Ambiental (APA), denominada APA de Santa Rita. A torre estava situada em $9^{\circ} 42^{\prime}$ $18^{\prime \prime} \mathrm{S}$ e $35^{\circ} 48^{\prime} 32$ " W, e ficou em operação no período de outubro de 2004 a setembro de 2005 . Os dados de Rd foram coletados com piranômetros CM5 da Kipp e Zonnen, os quais eram equipados com um anel de sombreamento de $10 \mathrm{~cm}$ de largura por $80 \mathrm{~cm}$ de diâmetro. Já para os dados de $\mathrm{Rg}$ utilizou-se o piranômetro SP - LITE da Campbell Scientific. Obteve-se também, por método empírico, o índice de transmissividade atmosférica $\mathrm{k}_{\mathrm{t}}\left(\mathrm{Rg} / \mathrm{R}_{\mathrm{o}}\right)$, onde $\mathrm{R}_{\mathrm{o}}$ é a radiação no topo da atmosfera. Os resultados mostraram uma grande variação entre as estações seca e chuvosa no tocante aos valores máximos alcançados pelo Rg, devido às variações das condições de transmissividade atmosférica. Internamente observou-se a grande dependência que a penetração dos raios solares tem do ângulo zenital solar (Z) com as estações do ano, onde se constatou uma redução de até $66 \%$ entre os períodos seco e chuvoso. Verificou-se também, que até $35 \%$ da radiação incidente na copa atingiu a superfície interna do mangue, e que esse valor reduziu-se para apenas $15 \%$ no período chuvoso.

Palavra Chave: Meteorologia, Radiação Solar, Índice de Transmissividade Atmosférica $\left(\mathrm{K}_{\mathrm{t}}\right)$ e Manguezais.
\end{abstract}

ABSTRACT: STUDY OF THE GLOBAL SOLAR RADIATION AND THE INTERNAL AND EXTERNAL TRANSMISSIVITY INDEX IN A MANGROVE FOREST IN ALAGOAS - BRAZIL. 
Mangrove forests are of extreme social, economic and environmental importance, because they sustain a rich fauna and flora. Brazil possesses one of the largest mangrove areas in the world, which extends from the extreme north to the southern regions of the country, covering the entire coast. Due to such vast expanse, altering the state of the mangrove forests has a direct influence on the microclimate of the region. From the meteorological perspective, it is necessary to limit the exploitation of this ecosystem because the destruction of the mangrove forests exposes the surface area to direct solar radiation, thus changing the total net radiation. Therefore, one of the prime factors in understanding the availability of energy to the diverse processes of the system is knowing how the Solar Global Radiation $(\mathrm{Rg})$ and its components, Diffuse Radiation $(\mathrm{Rd})$ and Direct Radiation $\left(\mathrm{R}_{\mathrm{D}}\right)$, behave in the forest. The main objective of this research was to evaluate and compare the behavior of the Rg and it components, inside and above the mangrove forest. The solar radiation measurements are from a micrometeorological tower installed inside a mangrove forest, located at the Marechal Deodoro municipal district (15 km from Maceió, Alagoas) in an environmentally protected area (APA) called APA de Santa Rita. The tower was situated at $9^{\circ} 42^{\prime} 18^{\prime \prime} \mathrm{S}$ and $35^{\circ} 48^{\prime} 32^{\prime \prime} \mathrm{W}$ coordinates, and operating from October 2004 to September 2005. The Rd data were collected with a CM5 Kipp and Zonnen pyranometer, which was equipped with a $10 \mathrm{~cm}$ width by $80 \mathrm{~cm}$ diameter shadow ring. A SP - LITE pyranometer from Campbell Scientific measured the Rg. The atmospheric transmissivity index $\mathrm{K}_{\mathbf{t}}$ $\left(\mathrm{Rg} / \mathrm{R}_{\mathrm{o}}\right)$ where $\mathrm{R}_{\mathrm{o}}$ is the top atmospheric radiation, was also obtained by an empiric method. The results showed great variation between the dry and rainy season relative to the maximum $\mathrm{Rg}$ value, due to the variation on the atmospheric transmissivity conditions. Inside the forest, it was observed that the penetration of the solar rays through the canopy is dependent upon the solar zenith angle $(\mathrm{Z})$, and a reduction up to $66 \%$ was eobserved between the dry and rainy seasons. It was also verified that up to $35 \%$ of incidental radiation at the top of the canopy reached the internal surface below the mangrove forest, reducing to only $15 \%$ during rainy season.

Keywords: Meteorology, Global Solar Radiation, Atmospheric Transmissivity Index $\left(\mathrm{K}_{\mathrm{t}}\right)$ and Mangroves.

\section{INTRODUÇÃO}

Manguezais são encontrados ao longo das costas tropicais e subtropicais e são considerados as únicas florestas que se desenvolvem em ambientes halófilos (Komiyama et al. 2008). As florestas de manguezais são de grande importância social, econômica e ambiental, por serem detentoras de uma rica estrutura peculiar que abriga uma grande variedade de plantas, animais e microorganismos, além de servir como berçário para várias outras espécies de seres vivos, que encontram nesse ambiente uma condição ideal para sua reprodução. Dessa forma, esses ecossistemas representam um patrimônio importante, na medida em que são consideradas zonas de elevada produtividade biológica e fonte de recursos pesqueiros (Souza e Sampaio, 2001). Parte dessa produtividade escoa para ecossistemas adjacentes, reciprocamente essas florestas também recebem materiais orgânicos advindos de estuários ou dos oceanos (Kristensen et al. 2008).

Atualmente uma das grandes questões ambientais diz respeito ao desmatamento das áreas de florestas nativas. Como em todo o mundo, no estado de Alagoas as áreas de mangue vêm sendo alvo de constantes pressões antrópicas (construção de aterros, recepção de dejetos, esgotos e produtos químicos diversos, loteamento, etc). A alteração do manguezal em área degradada altera os diversos processos, na interface atmosfera - vegetação, e conseqüentemente no microclima da floresta de mangue que, por conseguinte, afeta os processos ecológicos como a regeneração e o crescimento das plantas, a respiração do solo, ciclo de nutrientes e formação de habitat natural (Motzer, 2005). Dessa forma, no aspecto meteorológico, é necessário que seja limitada a forma de exploração dos manguezais, pois o calor transferido dos vegetais para a atmosfera provém da radiação solar, que incide sobre a superfície durante todo o período diurno. A retirada ou substituição da cobertura vegetal altera o balanço de radiação, principal fator de interação da superfície com a Camada Limite Atmosférica. Como a vegetação também armazena energia, modificações em sua cobertura resultam em uma maior ou menor disponibilidade de calor para os processos atmosféricos (Querino et al. 2006).

Radiação solar é toda radiação eletromagnética proveniente do Sol que atinge o planeta (Querino et al. 2006). Essa radiação é de extrema importância para a vida na Terra, pois é responsável pelos principais processos de ordem física, química e biológica, tanto animal quanto vegetal, bem como responsável direto na disposição da energia primária para todos os processos terrestres, desde a fotossíntese, até o desenvolvimento de 
tempestades, que provocam situações meteorológicas adversas (Souza et al. 2005). Então, o conhecimento do comportamento da Radiação Solar Global (Rg), formada pelas componentes direta e difusa, acima e no interior das florestas é primordial para o entendimento da disponibilidade de energia para os diversos processos desse sistema. A atenuação da radiação solar pela vegetação é um fenômeno físico e o conhecimento de seu perfil vertical no seu interior permite a definição da distribuição de energia disponível nos seus diferentes estratos e, por conseqüência, dos perfis verticais das fontes de calor e de vapor d'água (Marques Filho et al. 2005).

Outro importante fator nos modelos do balanço de radiação, em particular, modelos do clima, em geral, é a nebulosidade. As nuvens reduzem a quantidade de energia solar absorvida no sistema Terra-Atmosfera, tendo em vista que a refletividade das mesmas é bem maior do que a refletividade da atmosfera sem nuvens (Menezes e Dantas, 2002), e com isso há uma maior ou menor transmissão radiativa. Diversos estudos têm mostrado que esses modelos apresentam forte relação com o índice de claridade ou transmissividade $\left(\mathrm{K}_{\mathrm{t}}\right)$ (Wenxian et al. 1996, Riciere, 1998, Rensheng et al. 2004) e correntemente, tem sido feito uso do $\mathrm{K}$ para quantificar a $\mathrm{Rg}$ incidente em superfície horizontal (Meek, 1997).

Por sua vez, o $\mathrm{K}_{\mathrm{t}}$ ainda permite verificar a condição com que a radiação solar, após a passagem pela atmosfera, está alcançando a superfície. Ou seja, se mais diretamente ou mais espalhada, o que é fundamental para se compreender o montante de radiação que será disponibilizada na superfície das florestas. Salienta-se também, que esta mesma metodologia pode ser aplicada para a transmissividade através da floresta, o que permitirá compreender a extinção radiativa nesse sistema.
Apesar da grande importância do estudo dos manguezais sob vários aspectos da ciência, pouco se conhece a respeito dos processos de trocas de energia, dentro e acima do dossel desse tipo de floresta. Então, o principal objetivo deste trabalho é avaliar e comparar o comportamento da Radiação Solar Global $(\mathrm{Rg})$, dentro e acima de uma floresta de mangue, em função da transmissividade atmosférica e no interior da floresta.

\section{DADOS E METODOLOGIA}

\section{1 Área de estudo}

O Complexo Estuário Lagunar Mundaú-Mangaba compreende os Municípios de Maceió, Marechal Deodoro, Coqueiro Seco, Santa Luzia do Norte, Satuba, Pilar, abrangendo todas as ilhas e, no continente, as encostas dos Tabuleiros e Restingas que as circundam. A Área de Proteção Ambiental (APA) de Santa Rita, local onde foi instalado o experimento, ocupa uma área de 10.346.108 ha e situa-se entre as latitudes de $9^{\circ} 37^{\prime} 30^{\prime \prime}$ e $9^{\circ} 47^{\prime} 30$ "S e as longitudes $35^{\circ} 45^{\prime} 00^{\prime \prime}$ e $35^{\circ} 55^{\prime} 00^{\prime \prime} \mathrm{W}$, onde foi detectado que em 1985 a composição da APA era de 40,15\% de florestas naturais, 33,91\% de ocupações antrópicas e 25,31\% de superfícies líquidas, enquanto que em 2005 foi de 33,39\% de florestas naturais, 38,77\% de ocupações antrópicas e 25,20\% de superfícies líquidas (Nascimento et al. 2009).

$\mathrm{O}$ estudo foi realizado com a obtenção de dados provenientes de um sítio experimental localizado na ilha de Santa Rita no município de Marechal Deodoro, aproximadamente 15 $\mathrm{km}$ ao sul da cidade de Maceió - AL, durante o período de outubro de 2004 a setembro de 2005 (Figura 1). Essa floresta de mangue faz parte da área APA pertencente ao condomínio

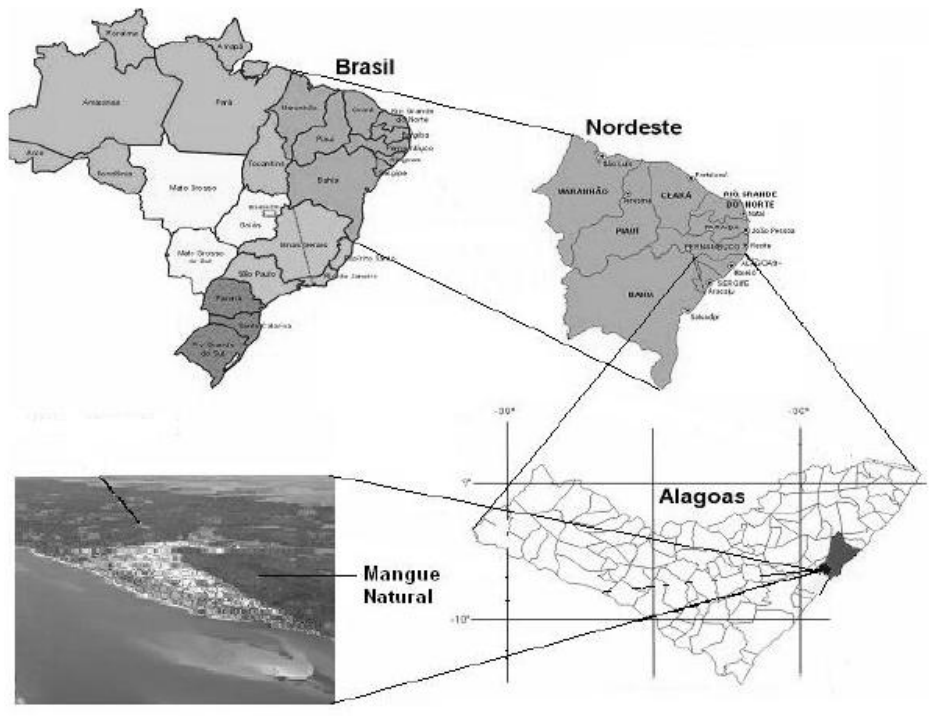

Figura 1 - Localização geográfica do Complexo Estuarino Lagunar Mundaú - Manguaba. 
Laguna, e está localizada a $9^{\circ} 42^{\prime} 18^{\prime}$ 'S e $35^{\circ} 48^{\prime} 32^{\prime \prime} \mathrm{W}$.

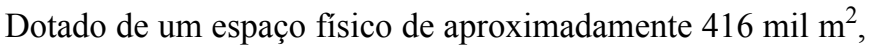
o manguezal tem uma flora composta por dois tipos de árvores predominantes, com uma altura média do dossel em torno de 11,5 m. Um dos tipos arbóreos é a Avicennia germinans L., vulgarmente chamada de mangue preto. Contudo, essa planta é encontrada em menor número do que a espécie Rhizophora Mangle L., também conhecida como Gaitera ou mangue vermelho. Esse vegetal se dispõe mais externamente nos manguezais, restringindo-se praticamente aos canais e ilhas (Calado e Sousa, 2002).

\subsection{Climatologia da região}

O estado de Alagoas está localizado em uma sub-região do Nordeste que possui grande variabilidade na distribuição pluviométrica devido a sua orografia e sistemas de ventos locais (Figueiredo, 2002). A normal climatológica para região segundo a Secretaria de Estado de Recursos Hídricos e Irrigação (SERHI), apresenta o período chuvoso compreendido entre os meses de abril e julho, enquanto o período seco está compreendido entre outubro e janeiro (Serhi, 2003). Os dados coletados durante o experimento condizem com a normal climatológica da região. Contudo, chama a atenção o fato dos meses mais chuvosos, maio e junho, terem sido bastante superiores em relação aos da normal, enquanto todos os outros meses ficaram com precipitação abaixo da média, destacando-se dezembro e janeiro que foram extremamente secos (Figura 2).

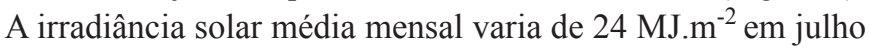
a $84 \mathrm{MJ} . \mathrm{m}^{-2}$ em novembro, assim como a temperatura média anual do ar varia de $19,3^{\circ} \mathrm{C}$ em agosto a $31,7^{\circ} \mathrm{C}$ em janeiro (Souza et al. 2005).

\subsection{Dados}

\subsubsection{Parâmetros mensurados}

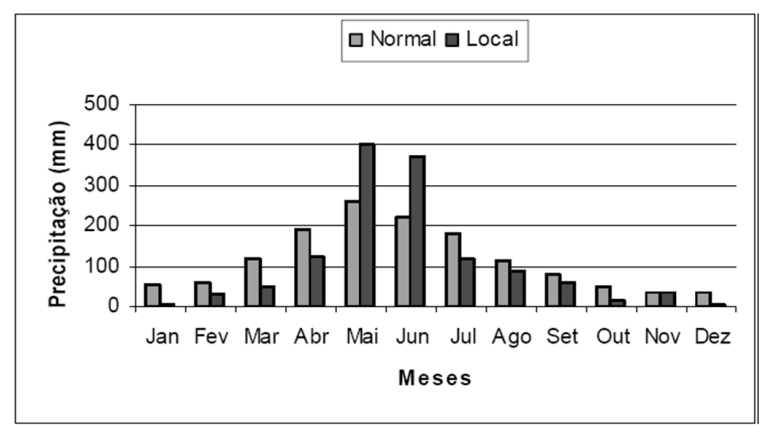

Figura 2 - Comparação entre a normal climatologia da precipitação (mm) para cidade de Maceió - AL e dados coletados na área degradada do experimento.
As medidas de Radiação Solar Global Externa (Rg_Ext), acima (13 m) e Radiação Solar Global Interna (Rg Int) no interior da vegetação $(2 \mathrm{~m})$, foram feitas por meio de dois piranômetros instalados em uma torre micrometeorológica, situada dentro do mangue. Os piranômetros destinados a mensurar a Rg_Ext e Rg_Int são do modelo SP - LITE da Campbell Scientific Inc., cujo tempo de resposta é inferior a um segundo, apresenta um desvio máximo estimado em $\pm 1 \%$ para cada $1000 \mathrm{~W} \cdot \mathrm{m}^{-2}$, e suas medidas encontram-se no intervalo espectral de 0,4 a 1,0 $\mu \mathrm{m}$. Os dados de Rd foram coletados com piranômetros CM5 da Kipp e Zonnen (intervalo espectral de 0,3 a 2,5 $\mu \mathrm{m}$, tempo de resposta de $30 \mathrm{~s}$ ), os quais eram equipados com um anel de sombreamento de $10 \mathrm{~cm}$ de largura por $80 \mathrm{~cm} \mathrm{de}$ diâmetro. Ressalta-se que ambos os sensores foram previamente testados e calibrados no Instituto de Ciências Atmosféricas da Universidade Federal de Alagoas - UFAL, antes de serem colocados em campo. Para dar uma melhor fundamentação na análise de nebulosidade, utilizou-se também, dados de insolação medidos na estação meteorológica de Maceió (n82994, Lat. $09^{\circ} 2$ ' $24^{\prime \prime} \mathrm{S}$ e $\left.35^{\circ} 14^{\prime} 24^{\prime \prime} \mathrm{W}\right)$.

\subsubsection{Parâmetros calculados e classificação da nebulosidade}

$\mathrm{O} \mathrm{K}_{\mathrm{t}}$ é definido como a razão entre $\mathrm{Rg}$ e a radiação solar que chega ao topo da atmosfera $\left(\mathrm{R}_{\mathrm{o}}\right)$ (Rensheng et al. 2004). Este índice irá variar de acordo com a quantidade de nuvens e aerossóis na atmosfera, e com isso haverá um aumento ou uma redução das componentes direta ou difusa, da radiação solar que atinge a superfície, possibilitando a classificação do céu quanto a sua nebulosidade (Tavares, 2005). Salienta-se que este índice é um número adimensional oriundo do quociente entre $\mathrm{Rg}$, e $\mathrm{R}_{\mathrm{o}}$ ambos em W.m ${ }^{-2}$.

Para uma apresentação mais detalhada da evolução diurna das componentes do balanço de radiação global, escolheram-se também quatro dias representativos de céu claro

Tabela 1 - Dias selecionados, representativos de diferentes condições de nebulosidade, de acordo com Kt

\begin{tabular}{ll} 
Dias & Índice de Claridade $\left(\boldsymbol{K}_{t}\right)$ \\
\hline $17 / 01$ & $\mathbf{K}_{\mathbf{t}} \geq \mathbf{0 . 7}(\mathbf{C L})$ \\
$09 / 02$ & $\mathbf{K}_{\mathbf{t}} \leq \mathbf{0 . 5}(\mathbf{P N})$ \\
$01 / 06$ & $\mathbf{K}_{\mathbf{t}} \geq \mathbf{0 . 7}(\mathbf{C L})$ \\
$31 / 05$ & $\mathbf{K}_{\mathbf{t}} \leq \mathbf{0 . 3}(\mathbf{N B})$
\end{tabular}

Kt $\leq 0,3$ - Dia Nublado (NB), 0,3<Kt $<0,7$ - Dia Parcialmente Nublado (PN) e $\mathrm{K} t \geq 0,7$ - Dia com Céu Limpo (CL). 
ou nublado, baseando-se no $\mathrm{K}_{\mathrm{t}}$ e adotando os critérios propostos por Iqbal (1983):

Os dias selecionados para representar diferentes condições de cobertura de nuvens, de acordo com o índice $K_{t}$, são listados na Tabela 1 .

Para estimativa da $\mathrm{R}_{\mathrm{o}}$, utilizou-se a expressão definida por Iqbal (1983) que é calculada em função da constante solar $\left(\mathrm{S}_{\mathrm{o}}=1367 \mathrm{~W} . \mathrm{m}^{-2}\right)$, raio vetor da órbita terrestre $\left(\mathrm{E}_{\mathrm{o}}\right)$, latitude local $(\varphi)$, declinação solar $(\delta)$ e ângulo zenital $(Z)$ :

$$
R O=S O E O \cos Z
$$

onde:

$$
E o=1+0.0033 \cos \left(\frac{2 \pi d_{n}}{365}\right)
$$

sendo $\mathrm{d}_{\mathrm{n}} \mathrm{o}$ dia do ano correspondente ao calendário Juliano. Para o ângulo zenital solar (Z), que consiste no ângulo entre o vetor que liga os centros da Terra e do Sol e o zênite local, utilizou-se a seguinte expressão:

$$
\cos Z=\operatorname{sen} \varphi \operatorname{sen} \delta+\cos \varphi \cos \delta \cos H
$$

em que, $\delta$ é a declinação solar que é considerada a latitude (celeste) em que se encontra o Sol, podendo variar de $0^{\circ} \mathrm{a} \pm 23^{\circ}$ ao longo do ano, e é calculada da seguinte maneira:

$$
\delta=23,45 \operatorname{sen}\left[\frac{360}{365}\left(284+d_{n}\right]\right.
$$

O ângulo horário (H), que é uma medida indicativa da posição do astro no sistema equatorial horário de coordenadas, assume valores entre $\pm 90^{\circ}, \mathrm{H}=0^{\circ}$ é atribuído ao meio-dia solar, enquanto valores negativos referem-se ao período anterior ao meio-dia solar e os positivos ao período posterior (Fattori e Ceballos, 2005). H é dado pela Equação 5:

$$
H=(\text { Hora }-12) 15
$$

\section{RESULTADOS E DISCUSSÕES}

A determinação e análise da Radiação Global no Topo da Atmosfera $\left(\mathrm{R}_{\mathrm{o}}\right)$ é essencial, uma vez que, através da comparação com a Rg à superfície, consegue-se caracterizar a qualidade da atmosfera com relação a sua turbidez, além de servir como suporte para os vários métodos empíricos de estimativa da radiação global na superfície.

Averigua-se que o ciclo médio horário anual de $\mathrm{R}_{\mathrm{o}}$ para a região de estudo obedece a sazonalidade, com uma variação de até $200 \mathrm{~W} \cdot \mathrm{m}^{-2}(15,3 \%)$ entre os períodos de ocorrência de maior e menor máximos (Figura 3). $\mathrm{A}_{\mathrm{o}}$ atinge o máximo de $1.300 \mathrm{~W} . \mathrm{m}^{-2}$ entre as 10:30 e 14 horas local, valores esses compreendidos entre setembro e meados de março e que coincide com a época seca da região. $\mathrm{O}$ menor pico máximo $\left(1.100\right.$ W.m $\left.{ }^{-2}\right)$ de $\mathrm{R}_{\mathrm{o}}$ ocorre no horário de 11 às $13 \mathrm{~h}$ nos meses entre abril e agosto, época em que o Sol localiza-se mais no Hemisfério Norte, e abrange toda a estação chuvosa regional.

Devido a importância para o desenvolvimento da vida na Terra, destaca-se a necessidade de conhecer o comportamento da $\mathrm{Rg}$, principalmente em áreas florestais, e neste caso em particular, em ambientes de manguezal que detém uma rica fauna e flora.

Destaca-se para a região em estudo um ciclo diário médio para Rg_Ext de 12 h, (Figura 4). Com isso o comportamento anual médio da curva de $\mathrm{Rg}$ Ext tende a acompanhar $\mathrm{R}_{\mathrm{o}}$. No entanto, os máximos médios são obviamente inferiores ao incidente no topo da atmosfera, apesar de coincidirem nos meses correspondentes. A explicação para tal redução seria o processo de atenuação sofrido pelos raios nas diversas camadas atmosféricas percorridas até atingir a superfície, ou ainda, segundo Gu et al. (2001), pode ser atribuído às reflexões múltiplas ocasionadas por camadas de nuvens, além também da absorção pelas mesmas.

Diante disso, percebe-se picos médios horários de 900 W. $\mathrm{m}^{-2}$ para Rg_Ext durante os meses compreendidos entre fim de setembro e março, para o intervalo das 10 às $14 \mathrm{~h}$, e outro variando de $\sim 600 \mathrm{~W} \cdot \mathrm{m}^{-2}$, para os meses de abril a julho (período chuvoso). O motivo para esses valores terem sido atingidos no intervalo de horas dos meses acima mencionado deve-se a fatores astronômicos. O caminho ótico a ser percorrido pelos raios solares provoca redução na quantidade de radiação que chega a superfície por conta de maior atenuação pela atmosfera, sendo menor nos horários próximos ao meio dia e,

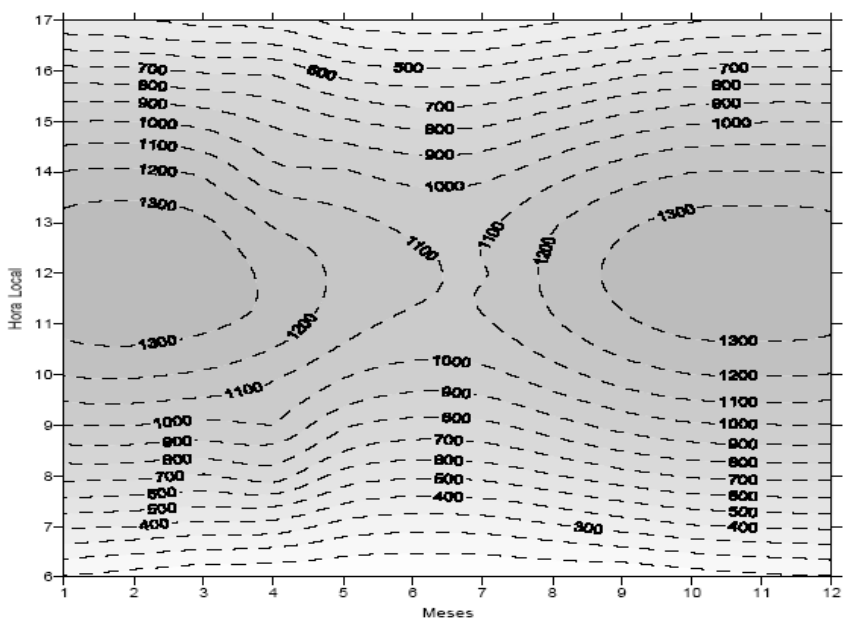

Figura 3 - Ciclo anual médio horário da Radiação Solar no topo da atmosfera (Ro) (W.m-2) no período de outubro 2004 a setembro de 2005 . 
por conseqüência, a incidência de radiação solar à superfície é mais próximo à perpendicular, provocando a chegada de mais energia. Esses máximos encontrados são muito próximos aos encontrados por Souza et al. (2005) em estudo sobre a Rg realizado na cidade de Maceió, distante aproximadamente 15 $\mathrm{km}$ do local do experimento. Os autores encontraram, em média, $886,03 \mathrm{~W} . \mathrm{m}^{-2}$ para o período seco e $630,19 \mathrm{~W} . \mathrm{m}^{-2}$ no chuvoso. Mesmo assim, nota-se que a distribuição média horária não é simétrica durante todo o ano. Percebe-se que o crescimento da curva é mais acentuado no período matutino, como também os máximos ocorrem aproximadamente às 11:30 h, o que estaria relacionado com o tempo solar verdadeiro, que para esta região apresenta uma defasagem média de 30 minutos.

$\mathrm{O}$ melhor entendimento para as variações de $\mathrm{Rg}$ relacionadas, tanto com à sazonalidade, quanto com às flutuações diárias, pode ser melhor compreendido ao observar o comportamento de $\mathrm{K}_{\mathrm{t}}$. Ao analisar a Figura 5, percebe-se que nos meses de verão no horário de máxima incidência solar $\mathrm{K}_{\mathrm{t}}$ $\geq 70 \%$, o que caracteriza uma transmissão atmosférica alta, implicando em uma quantidade mais abundante da radiação direta, e por conseqüência, com menos radiação difusa. Já nos meses de maio e junho tem-se uma transmitância média inferior a $60 \%$, assinalando-se uma atmosfera menos transparente, absorvendo, refletindo e espalhando mais radiação solar, o que resulta na redução da transmissão.

A diferença no ciclo diário pode ser facilmente compreendida verificando-se as alterações nas condições de turbidez atmosférica, que não só abrange aerossóis, como também nuvens. No período matutino, na época seca, tem-se $\mathrm{K}_{\mathrm{t}}$ maior que $60 \%$, sendo dessa forma característica de um céu mais transparente, o que permite que a radiação solar incidente seja menos espalhada na atmosfera e atinja a superfície mais rica em radiação direta. Já para o período vespertino o $\mathrm{K}_{\mathrm{t}}$ apresenta-se abaixo de $50 \%$. Isso já indicaria uma atmosfera

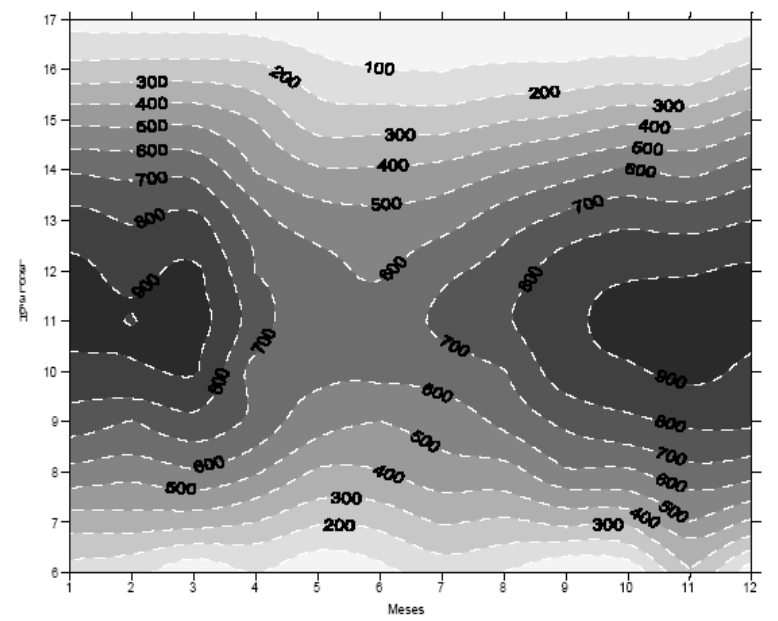

Figura 4 - Ciclo anual médio horário da Radiação Solar Global Externa (Rg_Ext) (W.m ${ }^{-2}$ ) no período de outubro 2004 a setembro de 2005. menos transparente, com uma maior atenuação da radiação impedindo que boa parte dela atinja a superfície. O motivo para essa redução, na parte da tarde, pode ser atribuído a uma maior concentração de aerossóis marinhos trazidos por ventos de Leste que segundo Dos Santos (2006), são mais intensos entre 10 e $14 \mathrm{~h}$, e que ao chegar à costa convergem para níveis superiores intensificando a formação de nuvens no período vespertino.

O fenômeno de precipitação além de influenciar nos vários processos de ordem física, química e biológica na superfície da Terra é responsável também pela limpeza da atmosfera, pois no ato da precipitação grande parte das partículas em suspensão retornam para a superfície deixando a atmosfera mais limpa.

Ao se verificar o comportamento anual da precipitação total horária acumulada (Figura 6), pode-se perceber que há maior intensidade no período de março a agosto, com maior ocorrência distribuída ao longo de três períodos distintos do dia: percebe-se o primeiro pico do total acumulado de precipitação ocorrendo durante toda a madrugada, isso provocaria a deposição de eventuais materiais particulados em suspensão, propiciando dessa forma, o aumento acentuado da Rg_Ext por conta de uma maior transmitância no início da manhã. Essa maior chegada de radiação no inicio da manhã, intensificaria a convecção matutina transportando umidade e conseqüentemente aerossóis da superfície para níveis superiores da atmosfera, implicando em um segundo pico entre as 11 e 12 h. Já o terceiro ocorre depois das $14 \mathrm{~h}$, onde são registrados os segundo maiores picos de acúmulos de chuva durante o dia, o que justifica redução na transferência de radiação solar através da atmosfera na maior parte da tarde, como pode ser observado na Figura 5. Durante todo o resto do ano, a precipitação é melhor distribuída durante o dia, com registros abaixo dos $3 \mathrm{~mm} \cdot \mathrm{h}^{-1}$ e chegando a não ocorrência de precipitação em algumas horas da época seca.

Para ilustrar as diferenças no ciclo diurno de Rg_Ext em dias de diferentes padrões de nebulosidade, apresenta-se

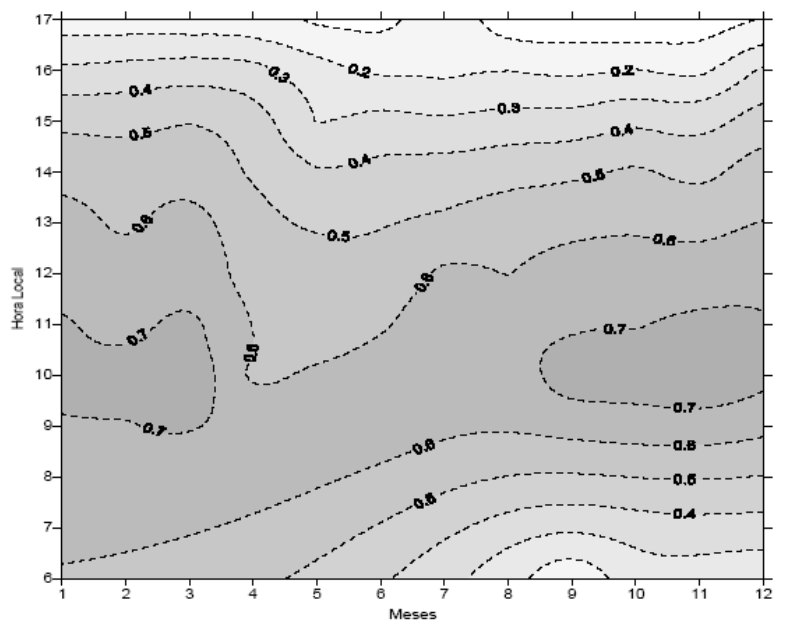

Figura 5 - Ciclo anual médio horário do Índice de Transmitância Atmosférica (Kt) no período de outubro 2004 a setembro de 2005. 


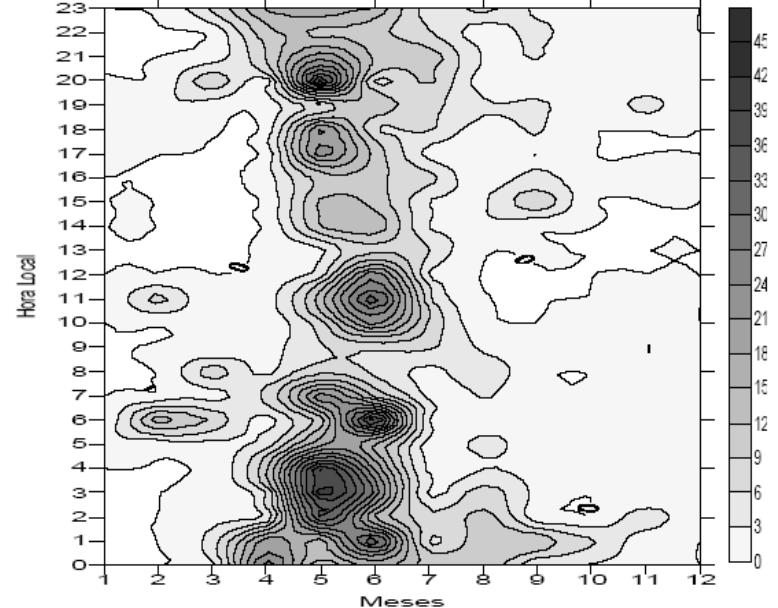

Figura 6 - Ciclo anual do total horário acumulado da precipitação (mm) no período de outubro 2004 a setembro de 2005.

na Figura 7 a evolução destas componentes ao longo dos dias selecionados na Tabela 1.

Verifica-se na Figura 7a (17/01), que se trata de um dia claro dentro da estação seca, comprovado pelo $\mathrm{K}_{\mathrm{t}}$ diário igual 0,7, implicando em uma configuração normal da curva Rg_Ext no decurso do dia, ou seja, sem a presença de oscilações, o que permite com que se atinja o pico de $1.000 \mathrm{~W} \cdot \mathrm{m}^{-2}$, fato comum para época que coincide com o verão. Quando se analisa um dia parcialmente nublado $K_{t}=0,5$ (Figura 7b) (dia 09/02), observa-se uma redução média de $17 \%$ da Rg_Ext. Ainda na
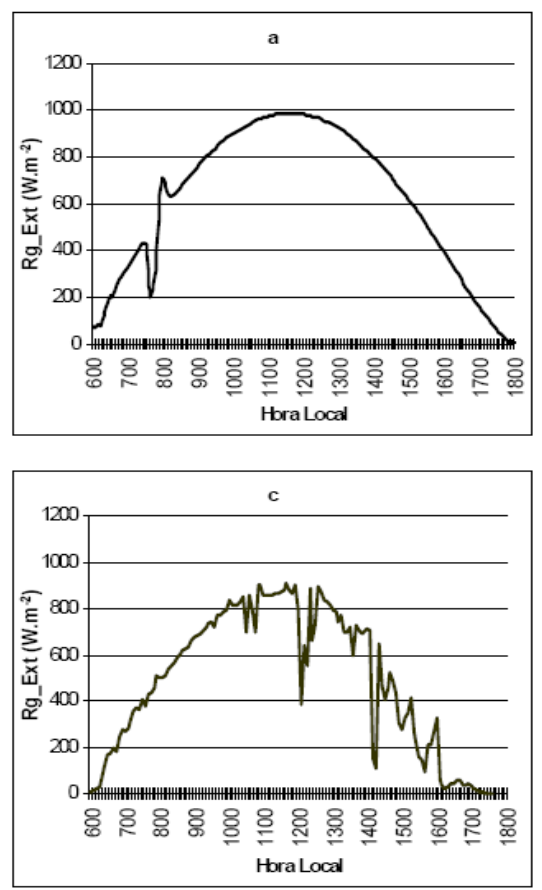

Figura $7 \mathrm{~b}$, percebe-se um declive abrupto da Rg_Ext, onde diminuiu de aproximadamente 1.200 para $200 \mathrm{~W} \cdot \mathrm{m}^{-2}$, o que significa uma redução de $83,4 \%$. A mesma situação pode ser notada para época chuvosa (Figuras $7 \mathrm{c}$ e $7 \mathrm{~d}$ ), com relação ao comportamento da $\mathrm{Rg}$ Ext em dias claros e nublados. O dia 01/06 (Figura 7c) apresentou $\mathrm{K}_{\mathrm{t}}=0,7$, o que o classifica como dia claro, enquanto o dia 31/05 (Figura 7d), apresentou um $\mathrm{K}_{\mathrm{t}}=0,3$ sendo dessa forma considerado dia nublado. Comparando-se os dias claros dos períodos seco e chuvoso, observa-se uma redução média de $20 \%$ no Rg_Ext na época chuvosa, devido à variação anual do ângulo de incidência da Rg_Ext, o que provoca uma variação no caminho ótico a ser percorrido pelos raios solares (Querino et al. 2006). Já os dias nublados apresentaram redução média de 50\%.

Em princípio, averigua-se que, assim como Rg_Ext, a Rg_Int apresenta um comportamento muito semelhante nos horários de ocorrência de máximos e mínimos além de obedecer a sazonalidade (Figura 8). Notam-se três diferentes picos de incidência de radiação solar na superfície do mangue ao longo do ano. Um máximo médio de $300 \mathrm{~W} \cdot \mathrm{m}^{-2}$ no período de novembro a março, que compreende a época seca e a fase de transição do período seco para o chuvoso. Nesse período, o menor ângulo zenital $(Z)$ da radiação solar, facilita a penetração por entre os galhos e folhas. Já no período chuvoso da região, entre o equinócio de outono e solstício de inverno no hemisfério sul, o registro máximo de Rg_Int foi de $100 \mathrm{~W} . \mathrm{m}^{-2}$, implicando em uma redução de $66 \%$ entre estes períodos extremos. O fato
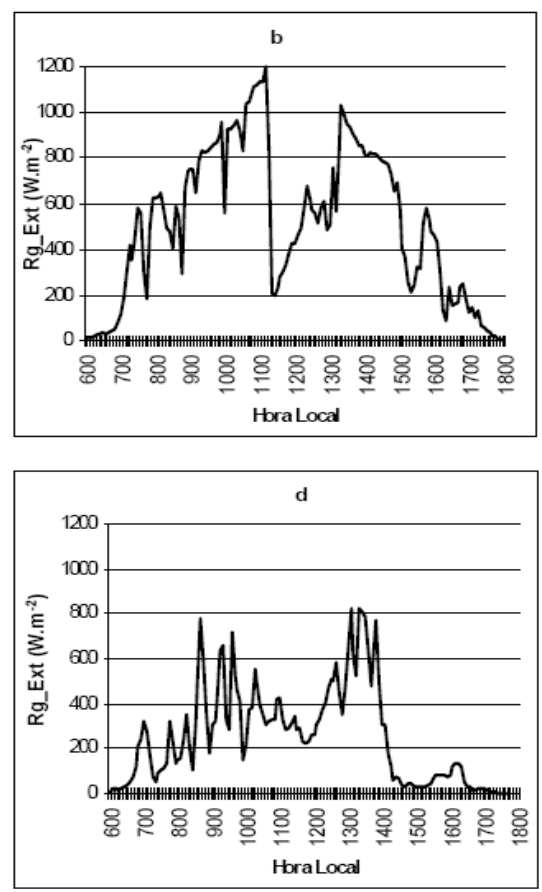

Figura 7 - Comportamento da Radiação Global Externa (Rg_Ext), (W.m² $)$, a cada 5min, na área de estudo nos dias; (a) 17 de janeiro, (b) 09 de fevereiro, (c) 01 de junho e (d) 31 de maio de 2005. 
provavelmente está relacionado a uma maior nebulosidade, pois conforme dados de insolação coletados na estação climatológica principal da cidade de Maceió, que registraram em média 6,4 h.dia ${ }^{-1}$, acredita-se que provavelmente boa parte dos dias encontrava-se com considerável cobertura de nuvens. Esse alto índice de cobertura de nuvens foi detectado também por Cabús (2002), que percebeu em seu estudo que mais de 60\% dos dias do ano encontram-se parcialmente nublados e apenas $12 \%$ são considerados dias claros. Essas nuvens tendem a espalhar e refletir a radiação de ondas curtas na atmosfera, proporcionando redução na quantidade que chega sobre a copa das árvores dificultando a penetração da radiação solar no dossel do mangue.

Os máximos médios entre 150 e $200 \mathrm{~W} . \mathrm{m}^{-2}$ nos meses de setembro e outubro, são decorrentes da transição entre a estação chuvosa para seca, e da passagem do Sol sobre o zênite local.

Ao se fazer a relação Rg_Int/Rg_Ext obtém-se a atenuação da radiação solar ao penetrar na vegetação ou a transmitância através do dossel da floresta. Verifica-se através da Figura 9, o comportamento anual dessa transmitância, que no início da manhã e no entardecer está abaixo dos $10 \%$, sendo os outros $90 \%$ atenuado, pelos galhos e folhas. No entanto, para às horas de incidência máxima, observa-se ao longo do ano três distintas variações, o que está de acordo com o Rg_Int. Nos meses compreendidos de dezembro a março, quando o Sol se encontra posicionado sobre o Hemisfério Sul, acontecem as menores atenuações, cerca de 70 a $75 \%$ não atinge a superfície do manguezal, ou seja, de 30 e $35 \%$, conseguem romper a barreira natural formada pelas árvores. Para os meses abrangentes da estação chuvosa (maio - agosto) encontra-se o registro das menores máximas médias transmitidas (apenas $15 \%$ ). Já para os meses de transição entre a estação chuvosa seca (setembro, outubro) e seca - chuvosa (fevereiro, março) a transmitância por entre o dossel foi entre 20 e $25 \%$, atenuando até $80 \%$ da Rg_Ext.

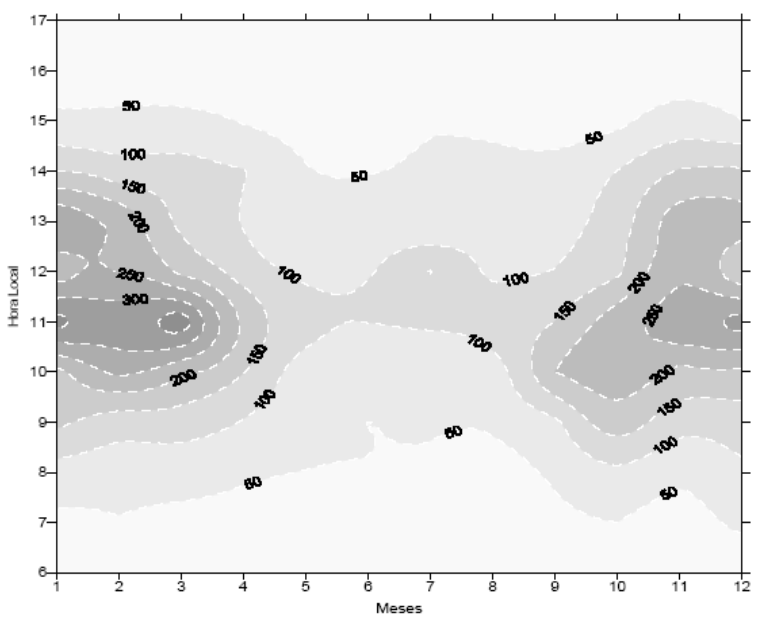

Figura 8 - Ciclo anual médio horário da Radiação Solar Global Interna (Rg_Int) (W.m ${ }^{-2}$ ) no período de outubro de 2004 a setembro de 2005.
As diferenças dos valores obtidos entre as estações do ano são atribuídas a dois fatores distintos, mas com mesma causa e efeito: o primeiro está relacionado com ângulo de incidência solar, pois de acordo com Hardy, et al. (2004), ele tem forte influência na interceptação e transmissão da radiação solar pelo dossel das árvores. O segundo é por conta da sazonalidade, pois como fora abordado anteriormente, essa redução implica também em uma diminuição na quantidade de $\mathrm{Rg}$ _Int.

Alguns autores têm realizado medições de radiação solar no interior de florestas, e o que se tem observado é uma discrepância de um tipo de floresta para outro (Shuttleworth et al. (1984), Schumacher (1992), Januário et al. (1992), Pereira et al.(2003), Pezzopane et al.(2000)).

As diferenças existentes para os diversos tipos de floresta estão relacionadas, de acordo com Hernandes et al. (2004), com estação do ano e com estádio fenológico característico de cada tipo de vegetação, principalmente quando se trata de plantas decíduas. A região de mangue aqui estudada, é caracterizada por árvores compostas de um longo tronco e uma camada de arbusto de aproximadamente $3 \mathrm{~m}$ de espessura no seu topo, sendo mantido um volume constante de folhas ao longo do ano. Não se nota dessa maneira, nenhuma variação das plantas com relação à sua fenologia, não nos permitindo atribuir esta variação de Rg_Int ao longo do ano a queda das folhas, mas sim a Rg_Ext e suas respectivas componentes (radiação direta e difusa).

Assim como realizado para Rg_Ext, tomou-se os mesmos dias para se analisarem casos isolados de Rg_Int em dias claros e nublados nas épocas seca e chuvosa (Figura 10). Na Figura 10a percebe-se que ao longo do dia existem dois horários (11:30 e 13:30 h) em que os valores de Rg_Int são superiores a $600 \mathrm{~W} \cdot \mathrm{m}^{-2}$. Para esses horários, os ângulos de incidência dos raios solares são os mesmos, o que leva a crer

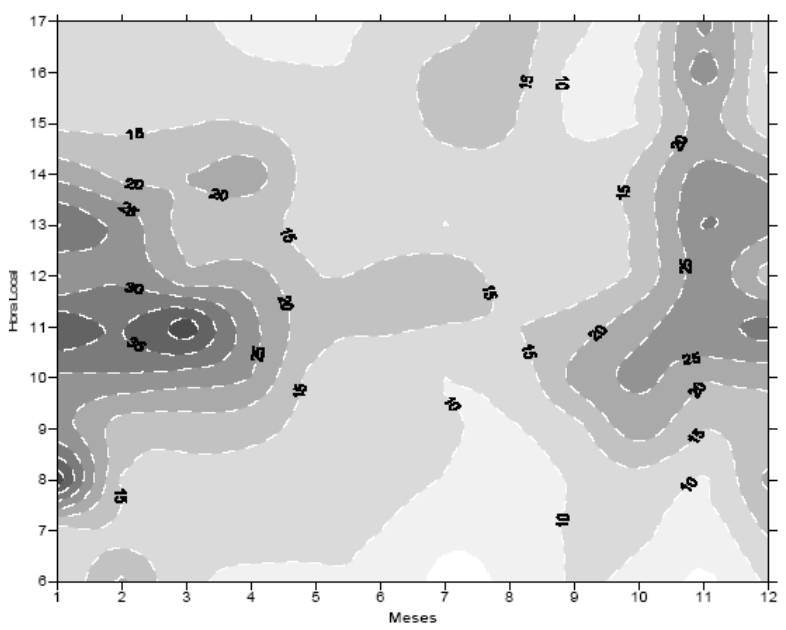

Figura 9 - Ciclo anual médio horário da razão (\%) entre Radiação Solar Global Interna (Rg_Int) e Radiação Solar Global Externa (Rg_Ext), no período experimental. 
que para essa inclinação angular existiam menos obstáculos até o instrumento. Salienta-se que esse registro é o equivalente a $60 \%$ da Rg_Ext atingindo a superfície interna do mangue, valor esse muito superior aos relatados por Geiger (1950) e Yoshino (1975) onde afirmam que em geral 80\% da radiação solar incidente é interceptada pelas copas das árvores e menos de $5 \%$ chega ao piso da floresta. Contudo, ressalta-se que essa estimativa não pode ser generalizada, pois os mesmos devem levar em consideração o tipo de florestas bem como suas características físicas, estação do ano e horário de incidência (Hernandes et al. 2004).

Notou-se na Figura $7 b$ que a $\mathrm{Rg}$ Ext sofreu uma forte oscilação em seu registro durante todo o dia 09/02. Esse desempenho da Rg_Ext implica em alteração no comportamento da Rg_Int (Figura 10b), onde a mesma, durante quase todo o período, não ultrapassou os $200 \mathrm{~W} \cdot \mathrm{m}^{-2}$. Contudo, em dois momentos foram registrados valores acima dos $400 \mathrm{~W} . \mathrm{m}^{-2}$, que coincidem com o horário da máxima incidência encontrada fora do mangue. Essa redução ocorre porque a penetração da radiação solar no dossel de florestas é fortemente dependente da incidência direta na copa das árvores, como o dia em questão é em grande parte nublado a parcialmente nublado, tem-se por conseqüência, mais radiação espalhada na atmosfera, o que acarreta redução na quantidade de radiação solar que atinge a copa do mangue. $\mathrm{O}$ reflexo dessa diminuição externa se faz notado nos fluxos internos onde se registrou 124,2 e 92,7 W.m ${ }^{-2}$ para dias claro e nublado respectivamente, implicando em uma redução de $25,6 \%$.

No dia 01/06 verifica-se uma redução de mais de 50\% no pico máximo de incidência da $\mathrm{Rg}$ _Int (Figura 10c), sendo registrado $360 \mathrm{~W} . \mathrm{m}^{-2}$, enquanto que o dia nublado da época seca, registrou valores superior a $700 \mathrm{~W} \cdot \mathrm{m}^{-2}$. Essa grande defasagem entre os períodos seco e chuvoso é também notada quando se analisa as médias diárias no interior do mangue. O valor de $\mathrm{Rg}$ _Int, na estação chuvosa, não ultrapassa os 60 W. $\mathrm{m}^{-2}$, encontrando-se para estação seca, uma média de 124 W. $\mathrm{m}^{-2}$, mostrando uma discrepância de $51 \%$ entre os dois dias, ocorrida por conta de uma maior inclinação de incidência dos raios solares, pois segundo Hardy et al. (2004), a interceptação e transferência da radiação entre o dossel de uma floresta, dependem, dentre outros fatores, do ângulo de incidência, uma vez que, quanto maior for esse ângulo, mais difícil se torna a entrada de radiação solar por entre os galhos.

Ao se estudar o desempenho Rg_Int no dia 31/05 (Figura $10 \mathrm{~d})$, verifica-se que sua média diária não ultrapassou os 30 W. $\mathrm{m}^{-2}$, chegando a ser cerca de $68 \%$ menor do que a média diária interna para o outro dia nublado em questão. Nota-se também, que após as 14 h, quando houve uma redução drástica da Rg_Ext, o registro de radiação dentro do mangue foi próximo de zero, provavelmente por conta de um maior espalhamento da Rg_Ext.

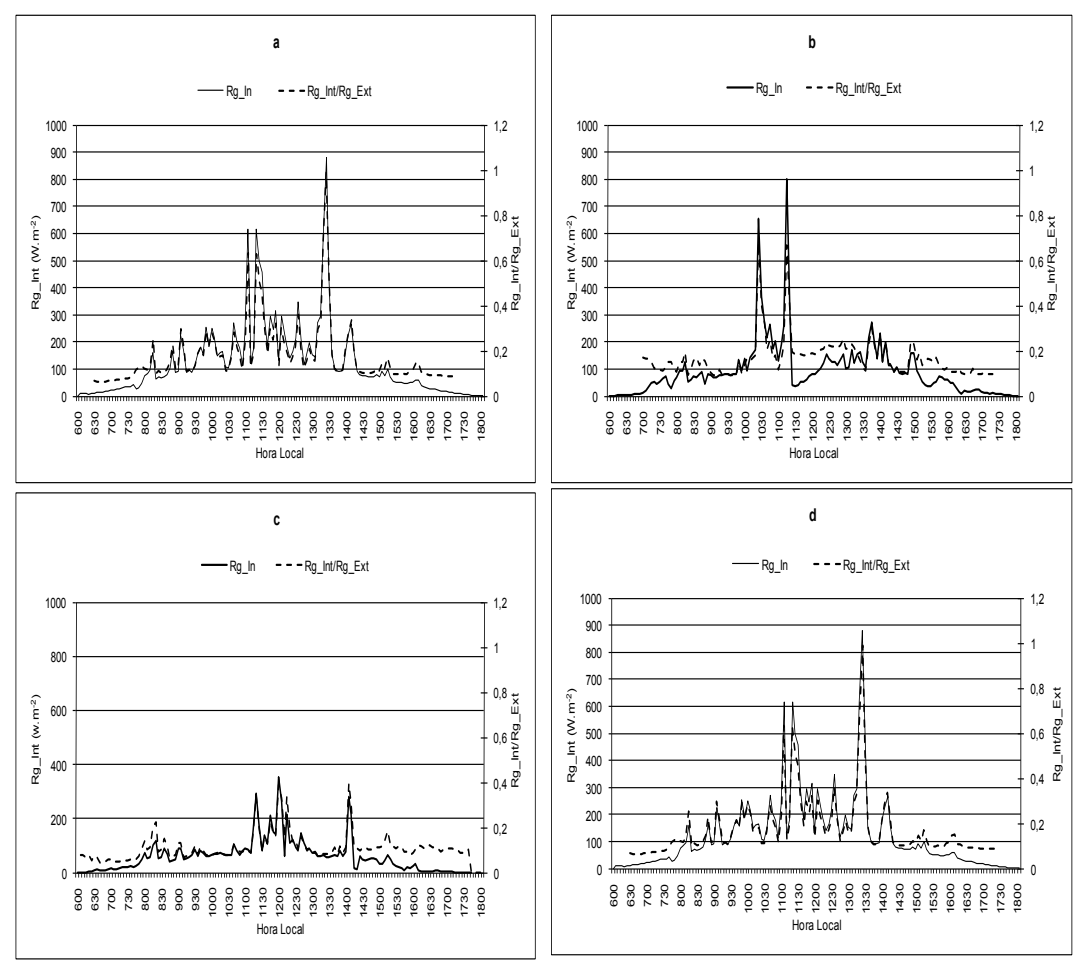

Figura 10 - Comportamento da Radiação Global Interna (Rg_Int), (W.m ${ }^{-2}$ ) e da relação Rg_Int/Rg_Ext,a cada 5min, sob a área de estudo, nos dias: (a) 17 de janeiro, (b) 09 de fevereiro, (c) 01 de junho e (d) 31 de maio de 2005. 


\section{CONCLUSÕES}

Com base nos resultados obtidos neste trabalho e as discussões a ele dedicadas, analisando-se a variabilidade da radiação solar incidente em área de floresta de mangue, enuncia-se as seguintes conclusões:

- Para Rg_Ext notou-se uma redução de 33\% na incidência média máxima entre as estações, além de se perceber um crescimento mais acentuado no período matutino. Comparando-se os dias claros dos períodos seco (entre outubro e janeiro) e chuvoso (abril até julho), observa-se uma redução média de 20\% no Rg_Ext na época chuvosa. Considerando-se dias nublados, observa-se uma redução ainda mais significativa (redução média de 50\%) da época seca para a época chuvosa.

- A Rg_Int sofre grande influência da nebulosidade, pois a mesma tende a espalhar mais radiação na atmosfera dificultando a penetração da radiação solar por entre os galhos, fato comprovado em análises relativas a dias claro e nublado. Percebeu-se também que Rg_Int apresentou forte dependência do Z, sendo as maiores médias mensais no período de menor ângulo de incidência, como também entre os períodos chuvoso e seco há uma redução de aproximadamente $66 \%$.

- A relação Rg_Int/Rg_Ext mostrou-se superior às encontradas para outros tipos de florestas tropicais, atribuído à estrutura física das árvores do mangue, que apresenta menor densidade no dossel, e a fatores como estação do ano e estádio fenológico característico de cada tipo de vegetação. Constatou-se também que essa relação é bastante influenciável pelo Z, apresentando maior atenuação nos períodos onde ocorre maior Z.

\section{REFERÊNCIAS BIBLIOGRÁFICAS}

CABÚS, R.C. Tropical daylighting: predicting sky types and interior illuminances in North - East Brazil. Reino Unido, 2002. Cáp.3, 14p. Tese (PhD em Arquitetura) - Universidade de Sheffield - School of Architectural Studies.

CALADO, T.C.S; SOUSA, C.E. Crustáceos do complexo estuarino - lagunar Mundaú/Manguaba Alagoas. Maceió: FAPEAL, 2002. 116p.

DOS SANTOS, T.V. Aspectos da distribuição temporal da precipitação e suas relações com as brisas na cidade de Maceió - AL. Maceió, 2006. 51p. TCC (Graduação em Meteorologia) - Instituto de Ciências Atmosféricas - UFAL.

FATTORI, P.A.; CEBALLOS, J,C. Glossário de termos técnicos em radiação atmosférica, 1997. Disponível na INTERNET via homepage http://tucupi.cptec.inpe.br/satelite/metsat/ pesquisa/gloss/capa.htm. Consultada no dia 19/07/2005.

FIGUEREIDO, J. O. de G. R. Distribuição espacial da pluviometria em Alagoas com ênfase à variabilidade causada pelos padrões de temperatura da superfície do mar. 2002, 148.p Dissertação (Mestrado em Meteorologia) - Universidade Federal de Alagoas - UFAL, Maceió, 2002. GEIGER, R. The climate near the ground. Cambridge, Inglaterra, 1950. $482 \mathrm{p}$.

GU, L.; FUENTES, J.D.; GARSTANG, M.; SILVA, J.T.; HEITZ, R.; SIGLER, J. Cloud modulation of surface solar irradiance at a pasture site in southern Brazil. Agric. Forest Meteorol., v. 106, p. 117 - 129, 2001.

HARDY, J.P.; MELLOH, R.; KOENING, G; MARKS, D; WINSTRAL, D; POMEROY, J.W.; LINK, T. Solar radiation transmission through conifer canopies. Agric. Forest Meteorol., v.126, p.257-270, 2004.

HERNANDES, J.L.; PEDRO JÚNIOR, M.J.; BARDIN, L. Variação estacional da radiação solar em ambiente externo e no interior de floresta semidecídua. Árvore., v.28, n.2, p. $167-172,2004$.

IQBAL, M. An introduction to solar radiation. Ed. AP. Vancouver Canadá. 389p, 1983.

JANUÁRIO, M.; VISWANADHAM,Y.; SENNA, R. C. Radiação solar total dentro e fora da floresta tropical úmida de terra firme (Tucuruí, Pará). Acta Amazônica., v. 22, p. 335-340, 1992.

KOMIYAMA, A., ONG J.E., POUNGPARN, S. Allometry, biomass, and productivity of mangrove forests: A review. Aquatic Botany v.89, p. 128-137, 2008.

KRISTENSEN, E., BOUILLON, S., DitTMAR, T., MARCHAND, C. Organic carbon dynamics in mangrove ecosystems: A review. Aquatic. Botany. v.89, p. 201-219, 2008.

MARQUES FILHO, A.O., DALLAROSA, R.G., PACHÊCO V.B. Radiação solar e distribuição vertical de área foliar Em floresta - reserva biológica do cuieiras - zf2, Manaus. Acta Amazônica, v. 35(4), p. 427 - 436, 2005.

MEEK, D.W. Estimation of maximum possible daily global solar radiation. Agricultural and Forest Meteorology. v.87, p. 223-241. 1997.

MENEZES, H.E.A.; DANTAS, R.T. Ajuste de funções para estimativa da irradiação solar difusa em Cajazeiras - PB. In: Congresso Brasileiro de Meteorologia, 12, 2002 Foz do Iguaçu - PR. Anais... 1 CD ROM.

MOTZER, T. Micrometeorological aspects of a tropical mountain forest. Agricultural and Forest Meteorology, v.135, p. 230-240, 2005.

NASCIMENTO, M. C.; SILVA, M. P.; GUIMARAES JUNIOR, S. A. M. Geoprocessamento aplicado a análise dos impactos ambientais na cobertura vegetal da Área de Proteção Ambiental de Santa Rita, Alagoas-Brasil.In: Anais XIV Simpósio Brasileiro de Sensoriamento Remoto, XIV, 2009, Natal - RN,Anais.... p. 4101-4108. 
PEREIRA, A.B.; VILLA NOVA, N.A.; GALVANI, E. Estimation of Global Solar Radiation Flux Density in Brazil from a Single Measurement at Solar Noon. Rev. Biosystems Engineering. v.86, n.1, p. 27-34, 2003.

PEZZOPANE, E.M.; RIBEIRO, A.; REIS, G.G.; REIS, M.G.F. Balanço de radiação em uma floresta em função da Declividade e Orientação das encostas. In: Congresso Brasileiro de Meteorologia, 11, 2000, Rio de Janeiro - RJ. Anais... 1 CD ROM.

QUERINO, C.A.S., MOURA, M.A.L., R.F.F. LYRA, MARIANO, G.L. Avaliação e comparação de radiação solar global e albedo com ângulo zenital na região Amazônica. Rev. Bras. de Meteorologia. v. 21(3a), p. 42 - 49, 2006.

RENSHENG, C.; ERSI, K.; JIANPING, Y.; SHIHUA, Z.W.; YONGJIAN, D. Estimation of Horizontal diffuse solar radiation with measured daily data in China. Renewable Energy. v.29, p. 717 - 726, 2004.

RICIERI, R.P. Modelos de estimativa e avaliação dos métodos de medida da radiação solar difusa. Botucatu, 1998, 81p. Tese (Doutorado em Ciências Agronômicas). Universidade Estadual Paulista "Julio de Mesquita Filho" Faculdade de Ciências Agronômicas.

SCHUMACHER, M. V. Aspectos da ciclagem de nutrientes e do microclima em talhões de Eucalytus camaldulensis Dehnh, E. grandis Hill ex Maiden e E. torelliana F. Muell. Piracicaba, 1992. 87p. Dissertação (Mestrado em Ciências Florestais) - Universidade de São Paulo - USP.
SERHI - Secretaria de Estado de Recursos Hídricos e Irrigação - Núcleo de Meteorologia e Recursos Hídricos de Alagoas - NMRG - AL, Posto: Maceió (01), 2003.

SHUTTLEWORTH, W. J.; GASH, J. H. C.; LLOYD, C. R. Observations of radiation exchange above and below Amazonian forest. Quart. J. R. Meteo. Soc., v.110, p. 1163-1169, 1984.

SOUZA, M. M. de A.; SAMPAIO, E.V.S.B. Variação temporal da estrutura dos bosques de mangue de Suape - PE após a construção do porto. Acta. Brasileira de Botânica, v.15(1), p. $1-12,2001$.

SOUZA, J.L. DE.; NICÁCIO, R.M.; MOURA, M.A.L. Global solar radiation measurements in Maceió, Brazil. Renewable Energy. v. 30, p. 1203 - 1220, 2005.

TAVARES, P. S. Observação e análise da radiação solar global e fotossinteticamente ativa na região de Maceió. 2005, 42p. TCC (Graduação em Meteorologia) - Universidade Federal de Alagoas - UFAL, Maceió.

WENXIAN, L.; ENRONG, L.; WENFENG, G.; SHAOXUAN, P.; TAO, T. Distribution patterns of diffuse solar radiation in Yunnan province, China. Energy Conversion and Management v. 37, p. 553-560, 1996.

YOSHINO, M. M. Climate in a small area: an introduction to local meteorology. Tokyo, Japão, 1975. 549 p. 